\title{
ASSESSING THE DRIVER'S RISK PERCEPTION DURING AUTONOMOUS DRIVING
}

\author{
C. Gandrez ${ }^{1,2, \otimes}$, F. Mantelet ${ }^{1}$, A. Aoussat ${ }^{1}$, F. Jeremie ${ }^{2}$ and E. Landel ${ }^{2}$ \\ ${ }^{1}$ Arts et Métiers ParisTech, France, ${ }^{2}$ Renault, France \\ $\triangle$ clara.gandrez@ensam.eu
}

\begin{abstract}
Advanced Driver-Assistance Systems were created to address the driver's failures. All these ADAS are a part of the evolution of the vehicles towards whole automation. To complete its launch in the automotive market, autonomous vehicles have to pass safety tests to acquire the consumers' trust. To receive the approval of the public, the self-driving car has to take into account the human feeling. The risk perceived by the driver is one of the new emotional form to integrate at the validation plan. The purpose of this study is to examine the perception of the risk of a self-driving car's driver.
\end{abstract}

Keywords: autonomous vehicle, risk feeling, cognitive capabilities, human behaviour, user experience

\section{Introduction}

Since the creation of the Anti-lock Braking System (ABS) in 1929, various advanced driver-assistance systems (ADAS) have been launched (European Commission, 2018). These systems were created to address the driver's failures but can also provide a certain comfort and raise efficiency. From the lane centering systems to park assist, all ADAS are a part of the evolution of the vehicles towards total automation. Self-driving cars would thus be the next evolution in the automotive market.

Autonomous vehicles would allow the driver to free himself from constraints linked to the driving task and drastically reduce road accidents, $94 \%$ of which are originating from a human failure (speed, alcohol, tiredness, inattention etc.) (Singh, 2015; Stein and Allen, 1987). Road safety would then be a reality.

At this time, many effective algorithms grant the driver optimal safety. Nevertheless, the driver being safe doesn't mean he feels safe. Having faith in a system can be a difficult task for the driver. Some actions the vehicle deems apparently safe could be considered worrying or hazardous by the driver. For example, many cases of anxiety attacks have already been identified among drivers using the "Park assist" function which helps the driver park the vehicle, with little to no intervention on his part. The vehicle can execute the action too quickly or abruptly for the driver which can frighten him. Taking into consideration the driver's feelings, comfort and safety became an essential challenge to solve before the launching of the autonomous vehicle.

The sense of security directly affects the acceptation of the self-driving car by the public. Taking into account the perceived risk sensation felt by the driver is done through the analysis of their emotions. This perception, subjective and unique for everyone, has already been explored and reviewed a long time ago. 
However, the emotional state of the driver has not been extensively studied in the case of autonomous driving.

The purpose of this study is to examine the perception of the risk of an autonomous car's driver and analyze the trust he can put in an automated system during worrying situations. A methodology of measure of the driver's emotional state is presented as well as the results of the driver's risk assessment.

\section{Literature review}

In this section, the notion of risk is defined. The parameters that influence the driver's risk perception are presented, and the public's acceptance of new technology, in particular self-driving cars, is detailed.

\subsection{Driver's risk perception}

Every living being can perceive a risk. Nevertheless, the feeling is subjective because it is a personal result of the evaluation of a situation: the feeling of risk depends on the subjective probability of an accident to occur as well as its subjective severity (Delignières, 1993). During the autonomous task, the perceived risk is a subjective experience of risk following the detection of potential danger in traffic (Elander et al., 1993).

Research on how drivers perceived the risk is essential to understand driving behavior and driving safety. Many studies have already analyzed the driver's risk perception using various assessment methods: surveys, driving simulators or conditioning participants with real world driving videos (Siren and Kjaer, 2014; Crundall et al., 2012; Borowsky et al., 2007). These researches on risk perception provided the means to define many characteristics impacting the driver's risk assessment. Principal factors are the gender of the driver (men and women don't evaluate risk the same way), their age, their driving experience (an experienced driver will have a better risk appreciation than a young driver) and their culture (Blasiis et al., 2017; Borowsky et al., 2007; Machado-Leon et al., 2016; Harris and Jenkins, 2006; Glendon et al., 1996; Lim et al., 2013).

However, in the case of an autonomous vehicle, its own characteristics play a role. The driver is not responsible of his actions anymore, meaning he doesn't choose the risk that the vehicle may decide to take. The perception of risk is attenuated if the risk is chosen voluntarily but amplified if it's imposed (Renn, 1992). Even though the risks might be similar, the voluntarily chosen risk is more acceptable than the imposed one meaning that the use of a self-driving car tends to amplify the perceived risks. Moreover, the control, or more precisely the lack of control, that the driver has on the vehicle also plays an important part. In a similar vein, risks perceived to be under one`s own control are more acceptable than risks perceived to be controlled by others (Schmidt, 2004). Another risk characteristic participates in the driver's risk assessment: habituation to the risk. A risk present for a long time is more accepted and its perception is attenuated due to habituation (even though the technical risk remains the same (Slovic et al., 1986)) whereas people are much more aware of unknown and new risks. Self-driving cars are a very new technology, unused by the public, which thus tends again to amplify the perceived risk. Finally, a last risk characteristic influences risk perception: the nature of the risk itself. It makes a great difference in risk perception if the risk or the actual damage is manmade or natural because the latter is more accepted than the former. This includes the control and voluntary aspects.

The profile of the driver (gender, age etc.) influences their risk perception. The risk perceived may be amplified by the use of an autonomous vehicle which includes many risk characteristics (control under the risk, habituation etc.). However, all these results had been studied for manual driving (or with slight automation) but have not yet been fully studied in the case of autonomous vehicles.

This risk perception is closely linked to the acceptance of new technologies.

\subsection{The self-driving car acceptance}

Relative to the nature of the risk and its familiarity, it is difficult for people to accept new technology without apprehension. In the automotive context, Osswald created in 2012 the "Car Technology Acceptance Research Model" (CTAM), a model that explains the acceptation (or rejection) process of advanced technology in vehicles based on the model of the "Unified Theories of Acceptance and Use of Technology" (UTAUT) (Venkatesh et al., 2003). This model also details the acceptation process but in a 
global way, tailored to no specific industry field. The Figure 1 shows Osswald's model. It highlights the importance of the perceived utility of the new technology for it to be accepted (Osswald et al., 2012).

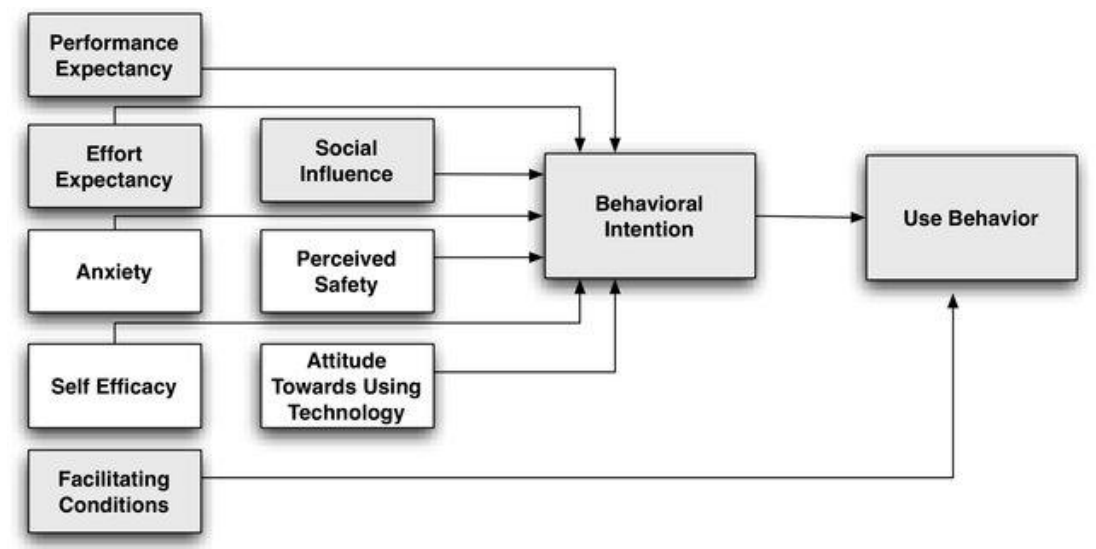

Figure 1. Car technology acceptance research model. Grey characteristics correspond to the UTAUT Model (Osswald et al., 2012)

The use of a new technology will be accepted if and only if this technology brings many benefits and may perform better than a human (Raue et al., 2019).

Taking into consideration the driver's risk perception could, according to this model, facilitate the public acceptation of autonomous vehicles.

\section{Research methodology}

Emotional reactions depend on the different Levels of Automation (LoA) as presented by the SAE J3016 in Figure 2 below. We assume an automation level of 3, meaning that the vehicle can act autonomously but the driver has to be attentive in order to take back control if it is necessary.

\section{LEVELS OF DRIVING AUTOMATION}

\begin{tabular}{|c|c|c|c|}
\hline Human driver monitors the driving environment & $\begin{array}{c}\text { The human driver } \\
\text { performs all the aspect } \\
\text { of the dynamic driving } \\
\text { task. }\end{array}$ & $\begin{array}{c}\text { The human driver } \\
\text { performs the driving } \\
\text { task with some driving } \\
\text { assist features }\end{array}$
\end{tabular}

Figure 2. SAE International Levels of Automation, 2018 (SAE, 2018)

To measure the emotional state of the driver and, in particular, the transition phase between a comfortable situation and a stressful one, we choose to evaluate the driver in a near-crash scene. Because evaluations on open roads are difficult to conduct, near-crash situations have been studied using digital simulations.

\subsection{Creation of the near-crashed scenario}

Scenarios were created with the comprehensive software SCANeR Studio. SCANeR is a software dedicated to automotive and transport simulation addressing both testing and driving for ADAS (Advanced Driver Assistance System), Autonomous vehicle, HMI and headlight use cases. It provides all tools and models that are necessary to build an ultra-realistic world: environment, vehicle dynamics, traffic, sensors, real or virtual drivers, weather conditions and scenario scripting (SCANER, 2018).

The scenario takes place on the highway, more precisely on the N104 stretch of road. A real road was used in order to increase the feeling of immersion in the scenario and its realism. The structure of the 
scenarios was defined as follows: the participant had to drive for about 3 minutes and when he heard a beep signal, he had to activate the autonomous mode with a button on the steering wheel. After this, the vehicle acts autonomously until the end of the scenario. One minute later, the traffic around the vehicle starts to become dense and the vehicle gets stuck in a traffic jam 2 minutes later. The nearcrash situation happens when the autonomous vehicle arrives in the traffic jam with the vehicle in front unexpectedly braking. The driver's confidence in the vehicle's actions was evaluated during this situation.

To measure how the driver perceives the risk and how it can be influenced by the environment, some parameters were different from one participant to another. Different scenarios were created; more precisely, 16 scenarios were defined thanks to an experience plan created using the Taguchi method (Constant, 1989). They are based on the previous storyline, in which 4 variables are studied: the effect of the weather (ideal conditions or a thick fog), the type of the preceding vehicle (motorbike, truck or car), the distance to the preceding vehicle when it brakes (very near, at the safety distance or far away) and the deceleration force when arriving in the traffic jam (from "very safe", with a value of $-0.2 \times 9.81$ $\mathrm{m} / \mathrm{s}^{2}$, to "quite abrupt", with a value of $\left.-0.5 \times 9.81 \mathrm{~m} / \mathrm{s}^{2}\right)$. Every scenario is the same, except for the 10 final seconds where the previous parameters differ.

The scenarios were then run on a dynamic driving simulator.

\subsection{The driving simulator}

To improve the realism of the perceived sensations, the Ultimate dynamic simulator, presented in Figure 3, was used.
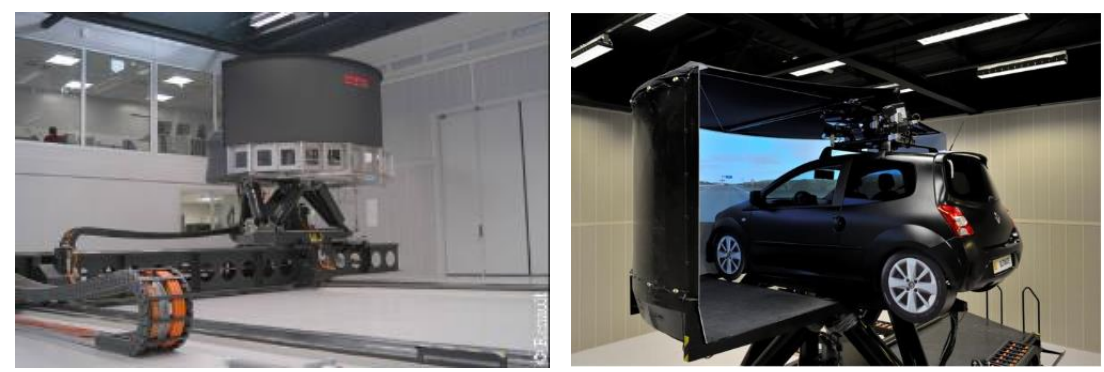

Figure 3. Ultimate dynamic simulator. On the left, the hexapod on the rails in a room allows for a realistic driving sensation. On the right, the cabin of the car mounted on the hexapod in front of the panoramic screen with a $210^{\circ}$ curvature

The Ultimate driving simulator is a high-level simulator, meaning that it was designed to create a credible driving experience despite the lack of faithfulness to the real. (Colombet et al., 2008). It is composed of a $210^{\circ}$-incurved high definition panoramic screen positioned to cover the whole driver's visual field. The notion of speed was mainly transmitted by the information detected in the driver's peripheral vision (Gabaude et al., 2019).

It is also composed of a "lightened" Twingo vehicle placed on six electromechanical actuators (hexapod). This hexapod can reproduce longitudinal acceleration movements (respectively deceleration) of up to $0.5 \times 9.81 \mathrm{~m} / \mathrm{s}^{2}$ (resp. $-0.5 \times 9.81 \mathrm{~m} / \mathrm{s}^{2}$ ), as well as sensations of roll, pitch and yaw identical to the movements inside a moving car. The hexapod stands on a deck capable of moving laterally or longitudinally on rails.

\subsection{Experimental procedure}

Each session of the experiment lasts for about 30 - 45 minutes and proceeds as follows.

Firstly, the participant is welcomed and must fill a survey with personal information in order to categorize which type of driver they are. In this survey, the participant could provide their gender, their age, their profession, the date of acquisition of their driver's license, the type of vehicle they use and the frequency of use (every day, sometimes a week, never etc.). An additional question asks the participant if they play video games. This question is asked in order to study the impact of playing video games on the participant's perception of realism of the simulation. 
After filling the survey, the participant is installed in the simulator. They are asked to drive approximatively 5 minutes inside the first scenario, which places them in an ideal case on a highway with no special events and normal conditions. This is done to familiarize them with the dynamics of the simulator. After that, the participant starts the near-crash scenario. At the beginning, they have to drive a few minutes and when a beep tone is heard, they have to activate the autonomous mode thanks to a button present on the steering wheel. Then, the vehicle acts autonomously for about 3 minutes without the driver input. However, the driver can take back the control of the vehicle if necessary, by braking or moving the steering wheel.

At the end of this scenario, a traffic jam is seen on the road. At this point, the driver has approximatively 3 seconds before the autonomous vehicle brakes.

Following this, the participant has to fill another survey with the help of an interviewer in order to detail each sensation that they felt and describe the situations they deemed stressful if there were any. They are asked if they would take control of the vehicle back when they see the traffic jam, why or why not, and if they felt safe during the braking phase. Then, they have to evaluate, using the following scale, how much they trusted the vehicle:

1. I fully trusted the vehicle

2. I trusted the vehicle

3. I had a few doubts about the capacities of the vehicle to handle the situation

4. I had strong doubts about the capacities of the vehicle to handle the situation

5. I had to take control of the vehicle immediately to avoid a crash

A Likert scale from 1 to 5 was chosen for the evaluation scale, associated with the previous verbatim. An uneven number of propositions were defined in order to provide the participants with a neutral choice if they didn't feel very safe or very insecure.

The two last questions of the survey were about the feeling of immersion of the participant in the simulation and if they found it realistic.

\subsection{Participants}

In order to study a specific driving style, all the participants were French. Moreover, driving style disparities exist even in France, thus the choice was made to select participants that came specifically from only one region which includes the capital and its surrounding areas.

Drivers were recruited via an internal tender sent to every department within Renault. Employees were invited to share this participation offer outside of the Technocentre (to their friends, family etc.) in order to attract people from outside the company that may have a naive vision of the autonomous vehicle.

In response to this tender, $89 \%$ of the 218 volunteers came from Renault's Technocentre and 22 came from outside. External participants consisted of various occupational backgrounds, such as retirees, nurses, accountants, unemployed people and two business leaders.

The panel was composed of 112 women and 106 men, ranging from 24 years old to 71 years old and distributed as shown below in Figure 4. The age distribution corresponds to the French driving population.

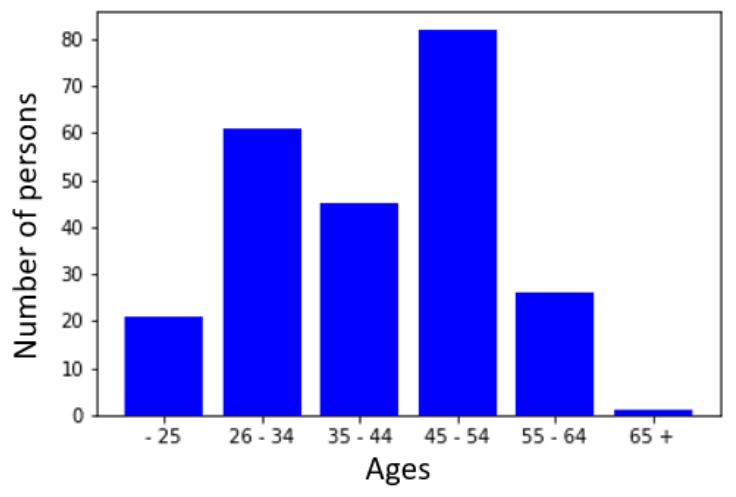

Figure 4. Distribution of the participants' ages 
Participants were classified in 3 categories of drivers: experts, intermediaries and novices based on their year of obtention of the driver's license and the frequency of use of their personal vehicle (every day, sometimes a week or a month etc.). A driver is considered an expert if they have held their driving licence for more than 10 years and if they drive every day. On the other hand, a driver is considered a novice if they never drive, only drive on rare occasions (several times a year or a month), or if they drive several times a week but have held their driving licence for less than 5 years. Other drivers are considered as intermediaries. Using the classification outlined in Table 1, 133 of them could be considered as experts, 53 as intermediaries and 32 as novices.

Table 1. Classification of a driver's experience based on the date of obtaining of their driving license and the frequency of use of their vehicle

\begin{tabular}{|c|c|c|c|}
\hline Everyday & $<\mathbf{5}$ years & Between $\mathbf{5}$ and $\mathbf{1 0}$ years & $>\mathbf{1 0}$ years \\
\hline Several times a week & Intermediaries & Intermediaries & Experts \\
\hline Several times a month & Novices & Intermediaries & Intermediaries \\
\hline Sometimes in a year & Novices & Novices & Novices \\
\hline Never & Novices & Novices & Novices \\
\hline
\end{tabular}

Participants were split into different scenarios. Each scenario was tested by 12 to 15 participants, with approximatively the same distribution of men/women, ages and driving skills.

\section{Results}

This section presents some results obtained from the experiments detailed in the previous section. In this section, the results observed from the notable differences between the participants for their risk perception and its assessment are described. A semantic analysis of the impact of fog, stopping distance, deceleration force and the type of the preceding vehicle on the risk assessment is also presented.

Experiments happened over four months, from July to October 2019. During that period, no autonomous vehicle crash was identified (the last accident before the experiments happened in March 2018). Moreover, no participants made any reference to it.

\subsection{Differences amongst the participants}

The distribution of external/internal participants could induce a bias given the fact that Renault employees could be better informed about how autonomous vehicle work and/or are familiar with the driving simulator. This population partially represents the global population because a large majority works in the automotive industry but come from diverse backgrounds (secretariat etc.) and finally few people were close to autonomous vehicles and even less had already seen or tested the simulator. Moreover, no significant differences were found when both groups (external or internal) were compared. These experiments were a first step, and the population selected for the next study will be increased and opened to international subjects.

Participants were not stressed by the test, but more than half felt impressed when they saw the simulator due to its massive appearance. This stress could be observed during the first driving test where the drivers did not feel comfortable with simulator dynamics. During this phase, the drivers drove more carefully than usual until they mastered the vehicle and adopted a more natural driving style (except for 9 drivers that took advantage of the fact that they were in a simulator in order to test its limits and exhibit behaviors more hazardous than their usual driving style). The habituation phase confirmed that it allowed the participant to relax and start the near-crash scenario feeling less stressed. However, approximatively $76 \%$ have been observed as anxious during activation of the autonomous driving mode but less than half were aware of this emotional state and described it during the interview.

From the notation on the scale of confidence, a significant difference between men and women is observed in their risk assessment ( $\mathrm{p}$-value $=0.00027495<0.001$ with a Fisher's Exact test. The difference in risk perception between men and women was already studied and demonstrated multiple 
times, but this difference can also be applied to the autonomous vehicle case. The gender of the driver influences their risk perception and their confidence in the autonomous vehicle.

In Figure 5 is presented the note attributed by the women (in light grey) and the men (in black).
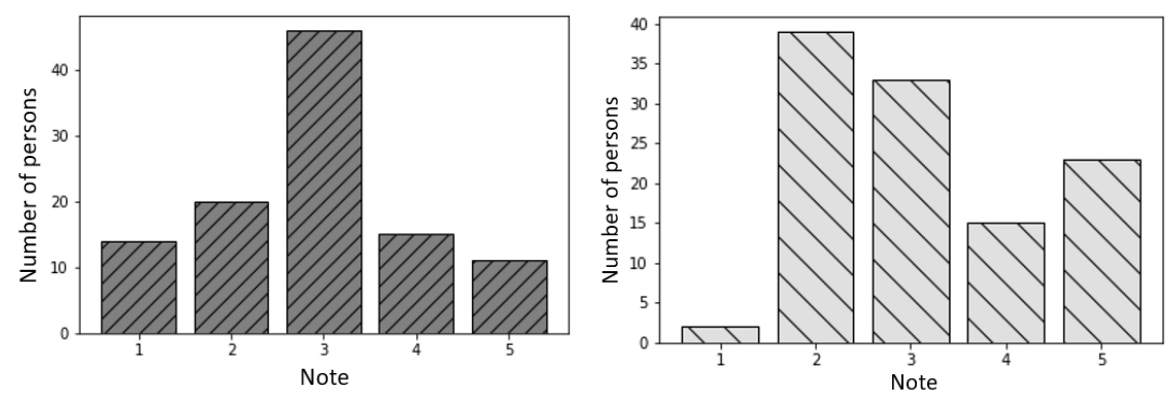

Figure 5. Risk assessment by the participants. On the left side, in black, men's risk assessment. On the right side, in light grey, women's risk assessment

By supplementing this result with the Kruskal-Wallis test, the fact that women have less trust in the autonomous vehicle, feel more anxious and have more doubts in its capacity to keep the driver safe can significantly be confirmed ( $\mathrm{p}$-value $=0.0008589<0.001$ ). Men feel less anxious but are more moderate in the trust given to the vehicle, $43 \%$ of them have just a few doubts at the beginning and want to test more worrying situations before having total faith in the system. Table 2 presents this result as well as the difference between the mean and the standard deviation of the note given by both groups. This result can be explained by the physiological difference between men and women, and the different hormones that their brain secretes during a stressful event.

Table 2. Presentation of mean and standard deviation of the note for the groups of men and women as well as the significant p-value of the Kruskal-Wallis test

\begin{tabular}{|c|c|c|c|}
\hline & Mean & STD & $\begin{array}{c}\text { p-value } \\
\text { (Kruskal-Wallis Test) }\end{array}$ \\
\hline Men & 2,8 & 1,12 & $<0,001$ \\
\hline Women & 3,2 & 1,16 & \\
\hline
\end{tabular}

Another observation relative to the risk assessment and the gender of the participant has been detected. This observation concerns the control of the vehicle. As presented in Table 3, women are more likely than men to take control of the vehicle back. This observation is related to the trust the driver has in the vehicle, which is less for women as seen previously.

Table 3. Presentation of the difference between men and women of number of people who retake (or don't retake) control of the vehicle

\begin{tabular}{|c|c|c|c|}
\hline Men & $\begin{array}{c}\text { Number of people who } \\
\text { retake control }\end{array}$ & $\begin{array}{c}\text { Number of people who } \\
\text { don't retake control }\end{array}$ & $\begin{array}{c}\text { p-value } \\
\text { (Fisher's Exact Test) }\end{array}$ \\
\hline Women & $64(60,4 \%)$ & $42(39,6 \%)$ & 0,04258 \\
\hline & $83(74,1 \%)$ & $29(25,9 \%)$ & \\
\hline
\end{tabular}

Another parameter influencing the trust placed in the vehicle by the driver is the participant's familiarity with driver assistance systems, especially with cruise control. A driver accustomed to cruise control felt less anxious than those who didn't use it (p-value $<0.05$ ). This is a new factor that influences risk perception compared to manual driving. This observation was already made on the German population (Brell et al., 2019). A self-driving car's driver's risk perception decreased as experience with driver assistance systems increased.

Nevertheless, only a weak correlation was noted between the level in driving and the trust placed in the vehicle globally and for each scenario (p-value $=0.1)$. The driving skills of the driver was attested as an influencing factor on his risk perception for manual driving but was not fully studied for the autonomous 
case. Risk assessment on self-driving cars seems to be different from manual driving because of a new factor influencing the perceived risk: a new technology with which few people are familiar and the lack of control on the vehicle. This observation does not seem to be aberrant given previous studies on autonomous vehicles which underlined that driving experience does not have a significant impact on the reaction time to take back control of the vehicle. These results are, according to the authors, also explained by the fact that the participants were not exposed to automated systems. Likewise, they showed that for an automation level higher than 2, driving experience does not have a significant impact on risk perception, in particular when the system's action was predictable (Sassman et al., 2018).

\subsection{Preceding vehicle type effect}

Three different types of preceding vehicles were involved: a car, a motorbike and a truck. A number of 180 persons had a car as preceding vehicle, 56 a motorbike and 58 a truck.

Only $11.5 \%$ of the participants decided to overtake the vehicle in front of them. Among these participants, twice as many preferred to overtake a truck or a car rather than a motorbike.

During the braking phase, when the autonomous vehicle reaches the traffic jam, another risk perception difference between a truck and a motorbike can be observed. For the hard-braking scenarios (deceleration lower than $-0.3 \times 9.81 \mathrm{~m} / \mathrm{s}^{2}$ ), drivers with a truck preceding them perceived a risk for themselves and braked to avoid a crash that could be lethal. They retook control because they felt insecure.

Drivers with a motorbike had the same reaction under these scenarios, but for different reasons. They also retook control of the vehicle but they specified that they felt safe. They braked to avoid hurting the biker but felt confident in the vehicle's ability keep them safe.

In the same situation, but with different types of vehicles preceding the autonomous vehicle, drivers didn't perceive the same risk: with the motorbike they perceived a risk for others, whereas in the case of a truck, they perceived a risk for themselves.

\subsection{Distance and deceleration effect}

Four values of deceleration were studied, ranging from $-0.2 \times 9.81 \mathrm{~m} / \mathrm{s}^{2}$ to $-0.5 \times 9.81 \mathrm{~m} / \mathrm{s}^{2}$. According to the participants in scenarios with $-0.2 \mathrm{~g}$ and where the safety distance was respected, $61.5 \%$ of them felt confident or very confident in the autonomous vehicle, and only $28 \%$ had a few doubts about the vehicle's ability to handle the situation. Other drivers felt insecure or had strong doubts in the vehicle when the distance with the preceding vehicle was under 2 meters and thought the vehicle wouldn't stop, causing them to manually brake.

For strong and very strong decelerations, participants declared that they perceived a high risk of collision and had to retake control of the vehicle ( $82 \%$ of the participants). Only $15 \%$ of them felt confident in the vehicle. The main reasons invoked in spite of these abrupt decelerations were that the participants knew how the autonomous algorithms work and how they are validated and they felt very confident in the process. For others, a few doubts persisted because they were trying it for the first time and thought more trust could be acquired with time.

For the last type of scenario, consisting in a deceleration of $-0.3 \times 9.81 \mathrm{~m} / \mathrm{s}^{2}$, the participants' feelings were divided. Half of them felt secure and confident, and the other half had a few doubts in the vehicle. Similarly to previous declarations, the principal reason invoked was the time it takes to get used to the autonomous mode, to see how it reacts and how drivers can trust it. For the drivers who had trusted the vehicle, a effect was observed. A major part of these drivers already used the cruise control option (or adaptive cruise control option) on their vehicles and declared that the reaction of the autonomous vehicle is similar to it.

The stopping distance of the autonomous vehicle, unless it was under the security threshold of 3 meters, was not pointed out by the participants. For scenarios with a low stopping distance, most of the participants braked for the vehicle, because they had the sensation that "the car wouldn't stop".

\subsection{Fog effect}

The simulation of a fog effect with a visibility range of 350 meters was applied to 106 participants. Among these participants, only $18.89 \%$ (20 participants) felt an apprehension to activate the autonomous mode under this weather condition. 
The principal reason for the participants to feel unsafe was a fear that the sensors would become defective or obstructed. Moreover, most of them felt "uncomfortable" with keeping the autonomous mode activated, and $10 \%$ of the participants affirmed that they "will never activate the autonomous mode in foggy conditions". Nevertheless, when a truck was preceding the autonomous vehicle, the participants felt less compelled to overtake it, due to the fog adding stress to the situation.

For the other participants, the fog was not a problem at all. Most of them thought that was an advantage to let the system drive because "sensors can see better than us" and "the system can react faster than a human". Furthermore, 2 participants felt very confident and reassured because they hadn't seen the traffic jam and about $13 \%$ felt "pleasantly surprised" because letting the vehicle drive "required less attention" on the driving task.

\section{Conclusion and discussion}

The purpose of this study was to determine which situations could be considered as worrying for an autonomous vehicle's driver and how they evaluated their risk perception. The experiments were performed on a dynamic simulator with 16 scenarios.

This study provides a better comprehension of how risk perception is assessed by an autonomous vehicle's driver, but this approach still needs improvements and extensions in following studies. New personal factors have to be considered compared to manual driving, such as the familiarity with driver assistance systems. Some results from the manual driving case could not be transposed to the selfdriving car, as the driving experience influences risk perception, due to new risk factors involved. For an experimented or a young driver, the confidence in an autonomous vehicle will rise over time.

Disregarding the driver's background, among the four factors studied that impact the perception of risk, the most relevant ones were the deceleration force, followed by the stopping distance if it is under the safety distance. On the other hand, the weather conditions, including reduced visibility, and the preceding vehicle type do not particularly affect the risk assessment for an autonomous vehicle's driver. Most of them consider the vehicle more performant than themselves.

These results must be treated with caution before comparing them to a real-case scenario. Even if the participants felt immersed in the simulation and even if the simulator transcribes the driving sensations as well as it could, a gap between real sensations and simulated sensations exists. Risks concerning the use of a real autonomous vehicle must be considered.

A limitation might be found in the fact that all participants came from France and in particular from only one region, but similar results were found in the neighbouring country of Germany. Moreover, a study showed that, similarly to manual driving studies, a generation's culture also influences the acceptance of autonomous vehicles (Lee et al., 2017). Results previously presented must be compared to several other countries (such as Italia, China etc.) which have different cultures to identify similarities and differences between populations. A logical next step is thus to conduct these researches amongst a larger population and with people from different locales.

Understanding how drivers assess risk and succeeding in determining in which emotional state is a self-driving car driver is essential to keep them confident in every situation and thus facilitate the public's acceptation of automation. Besides, being aware of the psychological state of the driver could be used to adapt the autonomous vehicle's driving style should the driver feel anxious or impatient. Automated functions can relax a driver that is detected as feeling stressed or annoyed in highly congested traffic, such as automatically starting his favorite music in order to put him in a calmer disposition and thus raise his level of comfort (Van der Zwaag et al., 2012).

\section{References}

Blasiis, M.D. et al. (2017), "Risk perception assessment using a driving simulator: a gender analysis", Conference Paper.

Borowsky, A., Shinar, D. and Oron-Gilad, T. (2007), “Age, skill, and hazard perception in driving”, Proceedings of the Fourth International Driving Symposium on Human Factors in Driver Assessment, Training and Vehicle Design. https://doi.org/10.1016/j.aap.2010.02.001

Brell, T., Philipsen, R. and Ziefle, M. (2019), "sCARy! Risk perceptions in autonomous driving: the influence of experience on perceived benefits and barriers", Risk Analysis, pp. 342-357. https://doi.org/10.1111/risa.13190 
Colombet, F. et al. (2008), "Motion cueing: What is the impact of the driver's behavior?", Proceedings of the Driving Simulation Conference.

Constant, C. (1989), "La méthodologie Taguchi: introduction à la recherche technologique - gestion fonctionnelle de la qualité ou gestion « off-line »", Revue de statistique appliquée, Vol. 37, pp. 47-77.

Crundall, D. et al. (2012), "Some hazards are more attractive than others: Drivers of varying experience respond differently to different types of hazard", Accident Analysis and Prevention, pp. 600-609. https://doi.org/ 10.1016/j.aap.2011.09.049

Delignières, D. (1993), "Risque préférentiel, risque perçu et prise de risque", Cognition et performance, pp. 79-102.

Elander, J., West, R. and French, D. (1993), "Behavioral correlates of individual differences in road-traffic crash risk: an examination of methods and findings", Psychol. Bull., pp. 279-294. https://doi.org/10.1037/00332909.113.2.279

European Commission (2018), Advanced Driver Assistance Systems, pp. 7-27.

Gabaude, C. et al. (2019), "Diagnostic et suivi des capacités visuo-attentionnelles des conducteurs âgés : Développement d'un programme de prévention".

Glendon, A.I. et al. (1996), "Age and Gender Differences in Perceived Accident Likelihood and Driver Competences”, Risk Analysis, Vol. 16 No. 5, pp. 755-762. https://doi.org/10.1111/j.1539-6924.1996.tb00826.x

Harris, C.R. and Jenkins, M. (2006), “Gender Differences in Risk Assessment: Why do Women Take Fewer Risks than Men?", Judgment and Decision Making, Vol. 1 No. 1.

Lee, C. et al. (2017), "Age differences in acceptance of self-driving cars: A survey of perceptions and attitudes", International Conference on Human Aspects of IT for the Aged Population, pp. 3-13. https://doi.org/ 10.1007/978-3-319-58530-7_1

Lim, P.C., Sheppard, E. and Crundall, D. (2013), “Cross-cultural effects on drivers' hazard perception”, Transportation Research Part F: Traffic Psychology and Behaviour, Vol. 21, pp. 194-206. https://doi.org/ 10.1016/j.trf.2013.09.016

Machado-Leon, J. et al. (2016), "Socio-economic and driving experience factors affecting drivers' perceptions of traffic crash risk", Transportation Research Part F: Traffic Psychology and Behaviour, pp. 41-51. https://doi.org/10.1016/j.trf.2015.11.010

Osswald, S. et al. (2012), "Predicting information technology usage in the car: towards a car technology acceptance Model", International Conference on Automotive User Interfaces and Interactive Vehicular Applications. http://doi.org/10.1145/2390256.2390264

Raue, M. et al. (2019), "The Influence of Feelings While Driving Regular Cars on the Perception and Acceptance of Self-Driving Cars", Risk Analysis, pp. 1-17. https://dx.doi.org/10.1111/risa.13267

Renn, O. (1992), "Concepts of risk: a classification", Krimsky S. and Golding D. (Eds.) Social theories of risk. Westport, CT. London: Praeger, pp. 53-79. https://doi.org/10.18419/opus-7248

SAE (2018), Sae international releases updated visual chart for its "levels of driving automation" standard for self-driving vehicles.

Sassman, M. et al. (2018), "Effets de l'automatisation de la conduite en situations de conduite normales et critiques: de l'étude empirique sur simulateur à la modélisation cognitive", ISTE OpenScience. https://doi.org/10. 21494/ISTE.OP.2018.0294

SCANeR (2018), https://www.avsimulation.fr/solutions/. [Online; accessed April-2019].

Singh, S. (2015), "Critical reasons for crashes investigated in the National Motor Vehicle Crash Causation Survey”, Traffic Safety Facts Crash Stats Report, № DOT HS 812115.

Siren, A. and Kjaer, M. (2014), "How is the older road users' perception of risk constructed?", Accident Analysis and Prevention, pp. 63-78. https://doi.org/10.1016/j.trf.2011.01.002

Schmidt M. (2004), Investigating risk perception: a short introduction, pp. 4-8.

Slovic, P., Fischhoff, B. and Liechtenstein, S. (1986), "The psychometric study of risk perceptions", Covello V.T., Menkes J and Mumpower J. (Eds.) Risk evaluation and management. New York, London: Plenum Press, pp. 3-24. https://doi.org/1794/22518

Stein, A.C. and Allen, R.W. (1987), "The effects of alcohol on driver decision making and risk taking", Alcohol, drugs and traffic safety-T86, pp. 177-181. https://doi.org/10.1016/0022-4375\%2887\%2990093-4

Van der Swaag, M. et al. (2012), "The influence of music on mood and performance while driving", Ergonomics, pp. 12-22. https://doi.org/10.1080/00140139.2011.638403

Venkatesh, V. et al. (2003), "User acceptance of information technology: Toward a unified view", MIS Quarterly, pp. 425-478. https://doi.org/10.2307/30036540 